\title{
Desarrollo y validación de la Escala de Mortalidad Neonatal-9 México para predecir la mortalidad en neonatos críticamente enfermos
}

\author{
Development and validation of the Neonatal Mortality Score-9 \\ Mexico to predict mortality in critically ill neonates
}

\author{
M. Cs. Horacio Márquez-Gonzáleza , Dra. María Valeria Jiménez-Báez \\ M. Cs. C. Mireya Muñoz-Ramírezc', M. Cs. Lucelli Yáñez-Gutiérreza, \\ Dra. Ana C. Huelgas-Plazad, Dr. Eduardo Almeida-Gutiérrez y \\ Dr. Antonio Rafael Villa-Romero ${ }^{f}$
}

a. Hospital de Cardiología, Centro Médico Nacional Siglo XXI, Instituto Mexicano del Seguro Social.

b. Coordinación de Investigación, Cancún, Quintana Roo.

c. Instituto Nacional de Pediatría.

d. Hospital de Ginecología y Obstetricia \#4 "Dr. Luis Castelazo Ayala".

e. Coordinación de Investigación en Salud, Instituto Mexicano del Seguro Social.

f. Departamento de Investigación de la Facultad de Medicina de la Universidad Nacional Autónoma de México.

Correspondencia: M. Cs. Horacio Márquez-González: horacioinvestigacion@ hotmail.com

Financiamiento:

Ninguno.

Conflicto de intereses:

Ninguno que declarar.

Recibido: 19-9-2014

Aceptado: 5-1-2015

\section{RESUMEN}

Introducción. Las escalas pronósticas son de utilidad para el médico que ejerce en las unidades de cuidados intensivos neonatales. Existen escalas neonatales validadas, en su mayoría para neonatos de bajo peso al nacer. El objetivo fue crear y validar una escala predictora de mortalidad en neonatos que incluyera nuevas variables pronósticas.

Población y métodos. Se realizó el estudio en un hospital materno-infantil dela ciudad de México, del Instituto Mexicano del Seguro Social. En la primera fase, se diseñó un estudio de casos y controles anidado en una cohorte (neonatos ingresados con criterios de gravedad durante el primer día de vida), en el que se identificó y construyó una escala con parámetros graduales de puntuación acumulativa de nueve variables independientes para predecir muerte: peso, acidemia metabólica, lactato, $\mathrm{paO}_{2} / \mathrm{FiO}_{2}, \mathrm{p}(\mathrm{A}-\mathrm{a})$ $\mathrm{O}_{2}, \mathrm{~A} / \mathrm{a}$, plaquetas y glucosa sérica. La validación se realizó en una cohorte prospectiva, de las mismas características, tomando como variable de desenlace la mortalidad hasta el séptimo día. Resultados. La cohorte incipiente estuvo conformada por 424 neonatos. Se seleccionaron 22 casos y 132 controles, y se identificaron 9 variables, que conformaron la escala nombrada escala de mortalidad neonatal-9 México. La cohorte de validación estuvo integrada por 227 neonatos. Se registraron 44 (19\%) defunciones, con un área bajo la curva de 0,92 . Con una puntuación de entre 16 y 18 , se reportó un hazard ratio de 85 (11-102), una especificidad de 99\%, un valor predictivo positivo de $71 \%$ y un valor predictivo negativo de $90 \%$.

Conclusiones. La escala propuesta es un instrumento fiable para predecir la gravedad en neonatos.

Palabras clave: mortalidad infantil, unidades de cuidado intensivo neonatal, factores de riesgo, índice de gravedad de la enfermedad.

http:/ /dx.doi.org/10.5546/aap.2015.213

\section{INTRODUCCIÓN}

La reducción de la muerte infantil en menores de 5 años es una de las metas del milenio. ${ }^{1}$
En promedio, se registran, en el mundo, cuatro millones de muertes durante las primeras cuatro semanas de vida. Las principales causas son prematurez, asfixia y sepsis. ${ }^{2} \mathrm{La}$ intervención con métodos preventivos ha logrado reducir hasta el $67 \%$ de las muertes en los neonatos. ${ }^{3}$

En México, ${ }^{4}$ en el año 2003, se registraron 2271700 muertes, de las cuales 20806 fueron en la etapa neonatal; el 49,4\% de ellas, por asfixia.

La detección de los neonatos críticamente enfermos ofrece la posibilidad de un tratamiento oportuno que impacte directamente en la supervivencia y en la disminución de la morbilidad. Las escalas pronósticas son de utilidad para el médico que ejerce en las unidades de cuidados intensivos neonatales (UCIN). Existen escalas internacionalmente utilizadas: ${ }^{5}$ SNAPPE $^{6}$ (Score for Neonatal Acute Physiology/Perinatal Extension), el CRIB II ${ }^{7}$ (Clinical Risk Index for Babies Score) y el NEOCOSUR (Neonatal del Cono Sur). ${ }^{8}$ En su mayoría, son empleadas en neonatos prematuros o con peso inferior a los $1500 \mathrm{~g}$.

Este estudio propone la construcción y validación de una escala predictora de mortalidad en neonatos críticamente enfermos, sin excluir a aquellos con peso superior a los 1500 g, a partir de la inclusión de nuevos factores pronósticos.

\section{POBLACIÓN Y MÉTODOS}

Con autorización del Comité Local 
de Investigación y Ética en Salud (R-2012-679089), se realizó el estudio en el Hospital General de Zona 2 A Troncoso, del Instituto Mexicano del Seguro Social (IMSS), en las siguientes fases:

a) Identificación de posibles variables de predicción: De enero de 2005 a diciembre de 2010, se conformó prospectivamente una cohorte, en la que se incluyeron neonatos de cualquier edad gestacional, de ambos sexos, embarazos múltiples, malformaciones externas aparentes; que fueran atendidos en la Unidad de Tococirugía del Hospital; ingresados a la UCIN durante el primer día de vida y que continuaron su atención hasta el egreso por cualquier causa. Se excluyeron pacientes nacidos en otras unidades y se eliminaron los neonatos egresados voluntariamente por sus padres o trasladados a otros hospitales. El cálculo del tamaño de muestra se fundamentó en la estadística del año previo, con un total de 3450 nacimientos y una mortalidad del $11 \%$, un $\alpha=95 \%$ y $d=99 \%(0,001)$, sustituidos en la fórmula de razón de proporciones para población finita, ${ }^{9}$ con un número de pacientes estimado de 204 . Se añadió un $10 \%$ estimado de pérdidas, con un cálculo final de 224 pacientes.

b) Asociación con la variable de resultado: Se investigaron variables prenatales (amenaza de parto pretérmino, infecciones prenatales, diabetes, preclampsia, ruptura prematura de membranas y administración de esteroides prenatales); perinatales (calificación Apgar y Silverman Andersen en el primer y quinto minuto, edad gestacional por Capurro en mayores de 32 semanas de gestación y Ballard en menores de 28 semanas, peso para la edad gestacional) y al ingreso en la UCIN (gasometría arterial, biometría hemática y química sanguínea tomadas durante la primera hora, índices ventilatorios: $\mathrm{p}(\mathrm{A}-\mathrm{a})$ $\left.\mathrm{O}_{2}, \mathrm{paO}_{2} / \mathrm{FiO}_{2}, \mathrm{~A} / \mathrm{a}\right)$. El tiempo inicial se consideró al ingresar a la UCIN. Se definió como variable de resultado la muerte hasta el día 7.

Se consideraron casos los pacientes fallecidos hasta el séptimo día y controles, todo paciente vivo hasta este punto. Se seleccionaron aleatoriamente 6 controles por cada caso (mediante el programa Aleator Method para Excel).

c) Evaluación de la capacidad de predicción en modelo independiente: Se realizó una asociación univariada de las variables de riesgo y las significativamente encontradas se sometieron a un análisis de regresión logística. Se identificaron nueve variables independientes (peso, Apgar al minuto 5, acidemia metabólica, lactato, gradiente y cociente alveolo-arterial, índice de Kirby y glucosa).

d) Construcción del puntaje de la escala: Las variables resultantes del modelo final se puntuaron basándose en la magnitud de la asociación estadística reportada por otros autores (artículos de pronóstico y metaanálisis). ${ }^{10-18}$ Los resultados se compararon con los de la primera cohorte: se consideraron como 0 puntos los valores reportados como normales y dentro de la mediana; 1 punto, a los correspondientes al tercer cuartil; y 2 puntos, al valor a partir del último cuartil (Tabla 1). La escala se nombró como Escala de Mortalidad Neonatal-9 México (EMN-9 Mex).

e) Cálculo de la precisión diagnóstica de la escala: Se sumaron las calificaciones individuales y se realizó una curva ROC para determinar el mejor punto de corte y calcular el área bajo la curva. La estratificación se eligió con los puntos correspondientes a los valores del 1sensibilidad de los siguientes rangos: < 0,5; 0,51-0,75; 0,76-0,94 y $\geq 0,95$.

f) Validación prospectiva de la escala: De enero de 2010 a diciembre de 2012, se diseñó una cohorte prospectiva de neonatos. Los criterios de selección fueron los mismos de la cohorte anterior. El inicio de seguimiento fue al momento del ingreso a la UCIN. El registro de las variables que conforman la escala fueron tomadas durante la primera hora de haber ingresado. Basado en la primera fase del estudio, en el cual de 424 pacientes atendidos en la UCIN se presentó una mortalidad del $5 \%$, un $\alpha=95 \%$ y d=99\% $(0,001)$, se calculó por razón de proporciones para población finita un tamaño de muestra de 90 neonatos. Se añadieron $10 \%$ de pérdidas y 10 pacientes por cada variable (90), lo que resultó en un tamaño final de 189 pacientes.

Análisis estadístico: para la estadística descriptiva, se emplearon medidas de tendencia central (medianas) y dispersión (rangos intercuartilares) en variables cuantitativas y frecuencias y porcentajes en variables cualitativas. En la primera cohorte, se calculó el riesgo por razón de posibilidades (OR: Odds Ratio) y se realizó un análisis de regresión logística. Para la segunda cohorte, se realizó un análisis de Cox y 
se calculó la razón de riesgo (HR: Hazard Ratio); análisis de supervivencia con Kaplan Meier y las pruebas de validez (sensibilidad, especificidad, valores predictivos, valores de verosimilitud y área bajo la curva) se realizaron para cada uno de los estratos propuestos.

El análisis se realizó en el paquete estadístico Statiscal Package for the Social Sciences (SPSS) versión 20 para Windows.

\section{RESULTADOS}

Primera cohorte: 424 neonatos completaron los criterios de selección. Se presentaron 22 casos y se eligieron aleatoriamente 132 controles (Figura 1.A.). Las diferencias entre los casos y controles, así como el cálculo y ajuste de los riesgos, se muestran en la Tabla 2.

Segunda cohorte: En el período de estudio, se atendieron un total de 7300 nacimientos, de los cuales 306 ameritaron manejo en la UCIN. Finalmente, se incluyeron 227 pacientes (Figura 1.B.). En promedio, la tasa de mortalidad neonatal temprana fue de 8,5 neonatos por cada 1000 nacimientos; 133 (58\%) se clasificaron con peso muy bajo para la edad gestacional.

De los participantes, 140 (61\%) fueron varones; la mediana de edad gestacional, de 33 (24-39) semanas; peso, de $1100 \mathrm{~g}$ (780-2100); y talla, de 43 cm (38-48). Se registraron 44 (19\%) defunciones.

El área bajo la curva de la escala fue de 0,92, $\mathrm{p}<0,0001$, para la predicción de mortalidad, $\mathrm{y}$ el HR de cada uno de los estratos ajustado por confusores se muestra en la Tabla 3. La validez de cada uno de los estratos se muestra en la Tabla 4.
La probabilidad de supervivencia acumulada al día 7 fue de $81 \%$. La Figura 2 muestra la supervivencia por estratos de puntuación.

\section{DISCUSIÓN}

Las escalas son una herramienta en la práctica médica para predecir el comportamiento de la enfermedad, alertar sobre el desarrollo de complicaciones o muerte, y para la subsecuente evaluación de programas de salud. ${ }^{19}$

El centro hospitalario donde se realizó el estudio se apega a las características poblacionales de la mayor parte de las ciudades. Las causas de las defunciones concuerdan con lo reportado en la epidemiología de México y del mundo. ${ }^{4}$

En la primera cohorte, se identificaron los factores asociados a la muerte y los resultados mostraron que las variables independientes en el modelo multicausal eran consistentes con las encontradas por otros autores, y algunas de estas eran ítems de escalas ya validadas. ${ }^{6}$ No obstante, otras, como los índices de ventilación y difusión, plaquetas e hiperglucemia, resultan novedosas comparadas con las escalas previamente mencionadas.

Las variables perinatales no demostraron utilidad en nuestro modelo pronóstico, fenómeno que ya ha sido contemplado por la escala SNAP (Score for Neonatal Acute Physiology) desde $2001,{ }^{20}$ pero que diverge de lo publicado por la NEOCOSUR, ${ }^{8}$ que incluye variables prenatales.

Es relevante mencionar que la administración de esteroides prenatales no representaron significancia en el modelo final, lo que discrepa

TABLA 1. Escala predictora de mortalidad en neonatos críticamente enfermos (EMN-9Mex)

\begin{tabular}{|c|c|c|c|}
\hline \multirow[t]{2}{*}{ Variable } & \multicolumn{3}{|c|}{ Puntaje } \\
\hline & 0 & 1 & 2 \\
\hline Escala Apgar al minuto 5 (puntos) & De 9 a 10 & De 7 a 8 & $\leq 6$ \\
\hline Peso al nacer (g) & $\geq 2500$ & De 1500 a 2499 & $\leq 1499$ \\
\hline Gasometría arterial $^{*}$ & Normal & Acidosis metabólica & $\begin{array}{c}\text { Acidosis } \\
\text { metabólica de } \\
\mathrm{BA}^{* *} \geq 15\end{array}$ \\
\hline Lactato (mmol/L) & $\leq 1$ & $1,1-3,9$ & $\geq 4$ \\
\hline $\mathrm{paO}_{2} / \mathrm{FiO}_{2}(\mathrm{~mm} / \mathrm{Hg})$ & $\geq 350$ & De 200 a 349 & $\leq 199$ \\
\hline $\mathrm{P}(\mathrm{A}-\mathrm{a}) \mathrm{O}_{2}(\mathrm{~mm} / \mathrm{Hg})$ & $\geq 100$ & 101-299 & $\geq 300$ \\
\hline $\mathrm{A} / \mathrm{a}$ & $\geq 0,6$ & $0,5-0,26$ & $\leq 0,25$ \\
\hline Plaquetas $(\mathrm{cel} / \mu \mathrm{l})$ & $\geq 150000$ & $50000-149000$ & $\leq 50000$ \\
\hline Glucosa sérica (mg/dl) & $\leq 126 \mathrm{mg} / \mathrm{dl}$ & De 127 a 200 mg/dl & $\geq 201 \mathrm{mg} / \mathrm{dl}$ \\
\hline
\end{tabular}

* Acidosis: $\mathrm{pH} \leq 7,28$ y exceso de base menor de $6 \mathrm{mmol} / \mathrm{L}$.

** Brecha aniónica (BA): $\mathrm{Na}-\left(\mathrm{HCO}_{3}+\mathrm{Cl}\right)$. 
de lo reportado por el grupo NEOCOSUR, ${ }^{8}$ probablemente porque la población no es cautiva en el centro sede del estudio y el tiempo necesario para la aplicación de inductores de madurez pulmonar es insuficiente.

La prematurez es la principal comorbilidad asociada a la muerte y queda representada en nuestra escala con el peso (por la posibilidad de multicolinealidad, se manejó en nuestra escala de esta forma), dada la conocida relación entre el bajo peso y la mortalidad..$^{21,22}$ Consideramos oportuno este resultado tomando en cuenta que la estimación de la edad gestacional puede perder fiabilidad para representar la prematurez, por la posible variabilidad en la interpretación de los instrumentos de clasificación, incluso entre pediatras. ${ }^{23}$

FIgURA 1. Esquema de selección de los integrantes de la primera cohorte (A) y segunda cohorte (B)

A

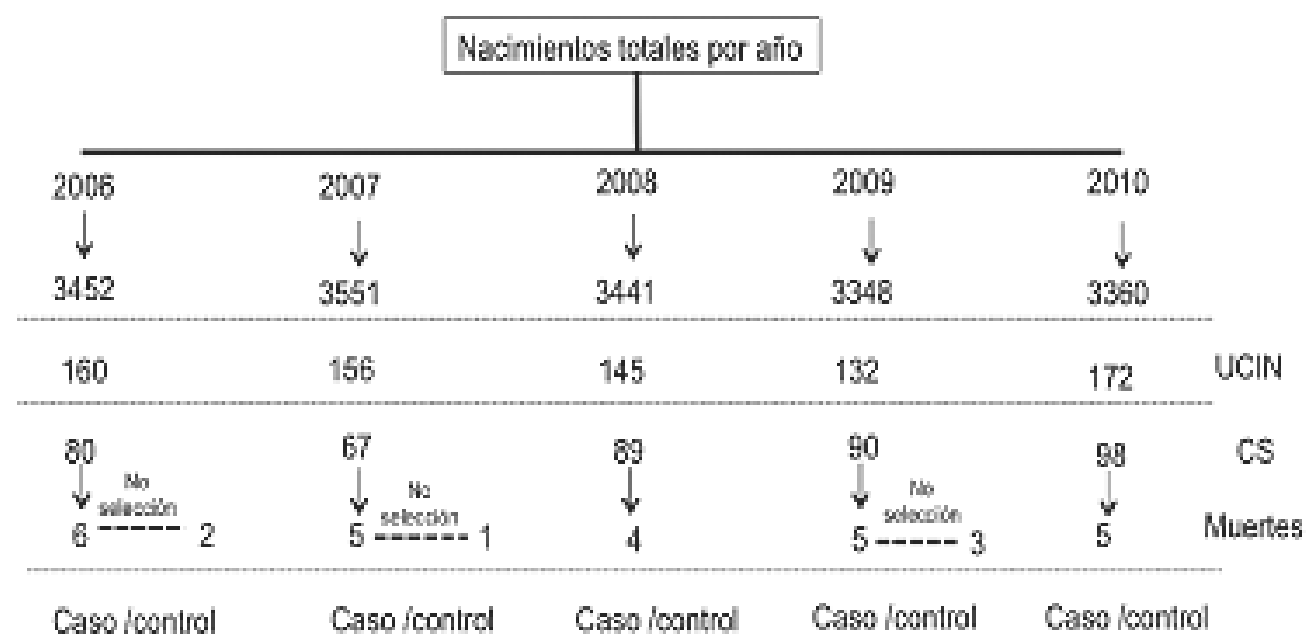

$4 / 24$

$4 / 24$

$4 / 24$

$5 / 30$

$5 / 30$

Tolal

22 casos y 132 controles

B

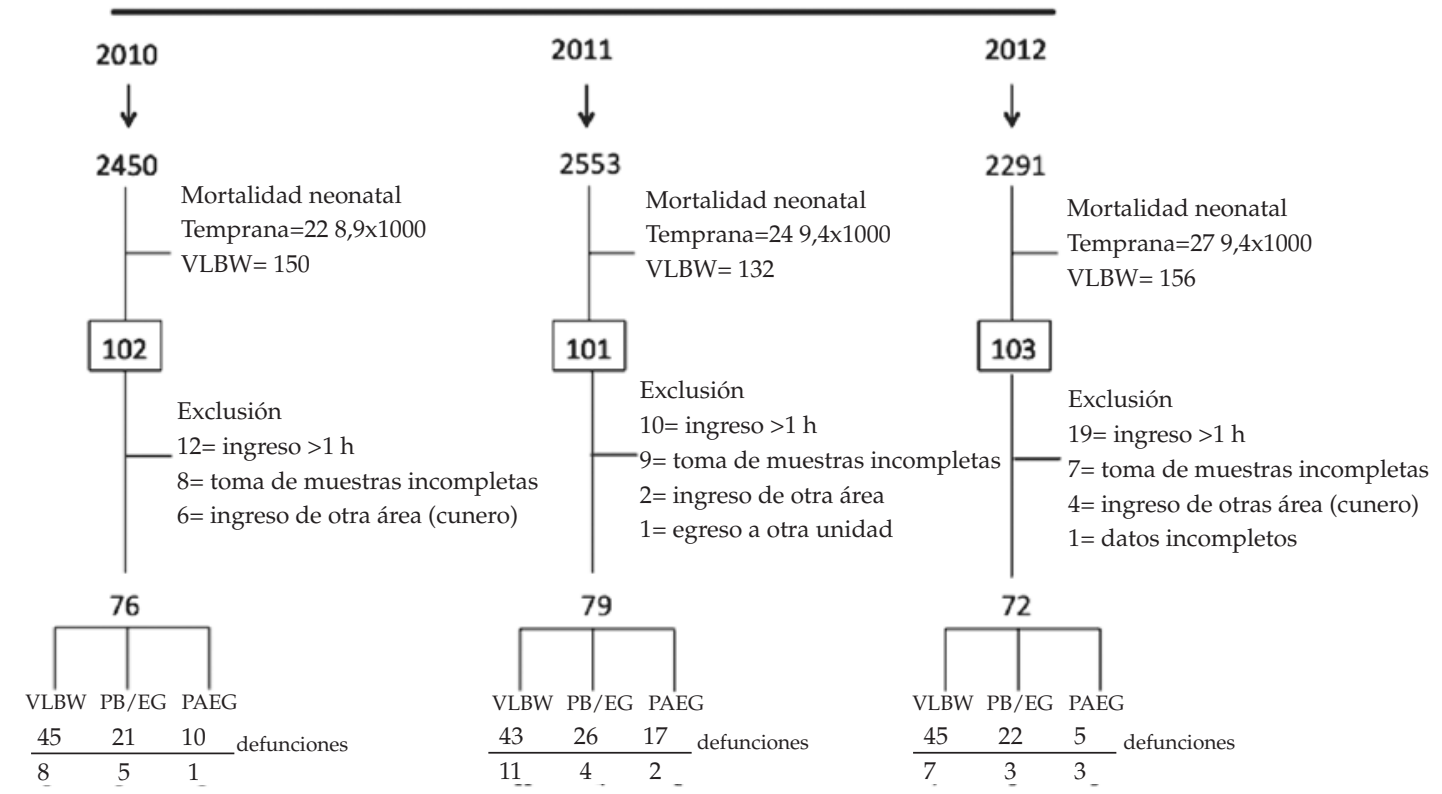

CS: completaron criterios de selección. VLBW: muy bajo peso al nacer.

PAEG: peso adecuado para la edad gestacional. PB/EG: peso bajo para la edad gestacional.

UCIN: Unidades de cuidados intensivos neonatales. 
La asfixia perinatal es una de las principales causas de muerte y es biológicamente plausible que el bajo puntaje de Apgar y la acidemia metabólica formen parte de la escala. ${ }^{24}$ Los problemas respiratorios, como el síndrome de dificultad respiratoria, la neumonía intrauterina o el síndrome de aspiración de meconio, son las principales comorbilidades y la gravedad de estas altera en distintos grados la difusión de oxígeno. El índice de $\mathrm{paO}_{2} / \mathrm{FiO}_{2}{ }^{25}$ infiere el daño pulmonar, mientras que el $\mathrm{p}(\mathrm{A}-\mathrm{a}) \mathrm{O}_{2}$ considera la diferencia de oxígeno que existe entre el alveolo y el capilar y el A/a calcula lo mismo sin estimar el efecto de $\mathrm{FiO}_{2}{ }^{26} \mathrm{El}$ resultado de ambas fórmulas interpreta la existencia de un trastorno a nivel de la membrana alveolo-capilar.
Los estados de hipoperfusión alteran los niveles de lactato, glucosa ${ }^{27}$ y la función plaquetaria. ${ }^{28}$ Considerando que la mayoría de las comorbilidades neonatales limitan la difusión de oxígeno tisular, se justifica que hayan mantenido independencia y formen parte de la escala.

Ninguna de las escalas previamente publicadas involucra la hiperglucemia como factor asociado a la mortalidad. Los niveles de glucosa se elevan debido a que existe una inmadurez en la secreción de insulina ${ }^{29}$ y se relacionan con patologías que ocasionan la muerte, como hemorragia intraventricular, sepsis, enterocolitis necrotizante, entre otras. ${ }^{30}$

La cohorte de validación del EMN-9 Mex resultó consistente con el grupo de pacientes de

TABLA 2. Resultados de la primera fase: comparación de factores de riesgo entre neonatos vivos y muertos

\begin{tabular}{|c|c|c|c|c|}
\hline Variable & Vivos $n=132$ & Muertos $n=22$ & $\begin{array}{c}\text { OR (IC-95\%) } \\
\text { crudo }\end{array}$ & $\begin{array}{c}\text { OR (IC-95\%) } \\
\text { ajustado }\end{array}$ \\
\hline Preclampsia & $21(16 \%)$ & $3(13 \%)$ & $0,7(0,1-3,7)$ & $0,8(0,3-19)$ \\
\hline Diabetes gestacional & $22(17 \%)$ & $6(25 \%)$ & $1,4(0,5-6)$ & $2(0,3-23)$ \\
\hline Malformaciones externas & $16(12 \%)$ & $4(20 \%)$ & $1,7(0,9-7)$ & $2,4(0,7-15)$ \\
\hline Amenaza de parto pretérmino & $9(7 \%)$ & $1(5 \%)$ & $0,8(0,09-6,9)$ & $0,4(0,2-5)$ \\
\hline Embarazos múltiples & $8(6 \%)$ & $1(5 \%)$ & $0,8(0,7-1,3)$ & $0,4(0,3-8)$ \\
\hline Infecciones en el tercer trimestre & $21(16 \%)$ & $3(13 \%)$ & $0,7(0,1-3)$ & $0,8(0,5-13)$ \\
\hline Ruptura prematura de membranas $>8 \mathrm{~h}$ & $25(19 \%)$ & $7(32 \%)$ & $1,7(0,5-5,8)$ & $1,3(0,4-14)$ \\
\hline Esteroides prenatales & $22(17 \%)$ & $1(5 \%)$ & $0,8(0,7-0,9)$ & $0,6(0,1-1,1)$ \\
\hline Apgar $<6$ en el primer minuto & $46(35 \%)$ & $15(70 \%)$ & $4(1,3-12)$ & $1,4(0,8-14)$ \\
\hline Apgar $<6$ en el minuto 5 & $12(9 \%)$ & $8(38 \%)$ & $5,9(1,8-19)$ & $3,5(1,7-5)$ \\
\hline Apgar $>3$ en el primer minuto & $30(23 \%)$ & $11(50 \%)$ & $3,1(1,1-9,2)$ & $14(0,4-20)$ \\
\hline Apgar $>3$ en el minuto 5 & $67(51 \%)$ & $15(69 \%)$ & $2(0,6-6,2)$ & $3(0,2-7)$ \\
\hline \multicolumn{5}{|l|}{ Edad gestacional } \\
\hline$>37$ semanas & $93(70 \%)$ & $4(18 \%)$ & $0,03(0,01-0,7)$ & - \\
\hline $32-36,6$ semanas & $30(23 \%)$ & $11(50 \%)$ & $2,3(1,2-7)$ & $1,2(0,4-1,9)$ \\
\hline$<31,6$ semanas & $9(7 \%)$ & $7(32 \%)$ & $3,1(1,7-8,9)$ & $1,5(0,9-12)$ \\
\hline \multicolumn{5}{|l|}{ Peso $(g)$} \\
\hline$>2500$ & $86(65 \%)$ & $5(22 \%)$ & $0,4(0,1-0,7)$ & - \\
\hline $1500-2499$ & $33(25 \%)$ & $7(33 \%)$ & $2,4(1,3-6,5)$ & - \\
\hline$<1499$ & $13(10 \%)$ & $10(45 \%)$ & $5(2,3-8)$ & $4,3(2-5,4)$ \\
\hline \multicolumn{5}{|l|}{ Sexo } \\
\hline Hombre & $53(40 \%)$ & $8(37 \%)$ & $1,01(0,9-1,1)$ & - \\
\hline Mujer & $79(60 \%)$ & $14(63 \%)$ & $0,9(0,3-2,2)$ & $0,5(0,3-13)$ \\
\hline Acidemia metabólica & $72(55 \%)$ & $20(91 \%)$ & $1,5(1,2-9)$ & $1,7(1,2-3,2)$ \\
\hline Brecha aniónica elevada (> 15) & $46(35 \%)$ & $31(70 \%)$ & $6(2-10)$ & $2,8(1,5-5,8)$ \\
\hline Lactato $>4 \mathrm{mmol} / \mathrm{L}$ & $33(25 \%)$ & $20(91 \%)$ & $23(6-310)$ & $12(3-17)$ \\
\hline Hemoglobina $<9 \mathrm{~g} / \mathrm{dL}$ & $5(4 \%)$ & $2(10 \%)$ & $1,3(0,6-5)$ & $1,1(0,5-1,5)$ \\
\hline Plaquetas < $100000 \mathrm{cel} / \mu \mathrm{L}$ & $9(7 \%)$ & $7(32 \%)$ & $5,4(1,5-18)$ & $2,9(2-4,3)$ \\
\hline $\mathrm{A} / \mathrm{a}<0,23$ & $66(50 \%)$ & $20(91 \%)$ & $1,7(1,1-1,5)$ & $1,3(1,1-2)$ \\
\hline $\mathrm{P}(\mathrm{A}-\mathrm{a}) \mathrm{O}_{2}$ & $59(45 \%)$ & $21(95 \%)$ & $1,8(1,2-2)$ & $1,4(1,2-3)$ \\
\hline $\mathrm{paO}_{2} / \mathrm{FiO}_{2}<200$ & $106(80 \%)$ & $21(95 \%)$ & $1,8(1,1-2,1)$ & $2,2(1,3-4)$ \\
\hline Hiperglucemia & $26(20 \%)$ & $11(50 \%)$ & $4(1,3-11)$ & $2,4(1,7-3,6)$ \\
\hline
\end{tabular}

Hiperglucemia: niveles por encima de $126 \mathrm{mg} / \mathrm{dl}$ en neonatos a término y $180 \mathrm{mg} / \mathrm{dl}$ en pacientes pretérmino.

Malformaciones externas: se refieren a las alteraciones mayores, como alteraciones de línea media, cardiopatías congénitas cianógenas graves, afecciones cefálicas o cualquier otra evidente que comprometa las funciones vitales.

Gradiente alveolo-arterial de oxígeno: $\mathrm{p}(\mathrm{A}-\mathrm{a}) \mathrm{O}_{2}$.

Cociente alveolo-arterial: $\mathrm{pAO}_{2} / \mathrm{paO}_{2}\left(\mathrm{pAO}_{2}\right.$ o presión arterial de oxígeno: $\left(\mathrm{PB}-\mathrm{P}_{\mathrm{H} 2 \mathrm{O}}\right) \times \mathrm{FiO}_{2}-\left(\mathrm{PaCO}_{2} / 0,8\right)$ x 1,25).

* El guión (-) representa la categoría de referencia.

OR: razón de probabilidades.

IC: intervalo de confianza. 
la cohorte original, en la que surgieron los ítems que conformaron esta escala. Todas las variables implicadas mantuvieron su significancia.

La mayoría de las escalas de mortalidad ponderan el peso de la predicción de sus variables mediante los coeficientes $\beta$. Resulta difícil sustituir estos valores durante la práctica habitual; por esta razón, se consideró que la construcción e interpretación de la EMN-9 Mex fuera fácil a partir de una técnica sumatoria, dado que los usuarios están habituados al uso de otras escalas similares, como el Apgar. ${ }^{31}$

Las escalas SNAP y SNAPPE son las más difundidas. En su primera versión, incluyeron una amplia gama de variables que complicaron su aplicación, por lo que se motivó una reedición

TABLA 3. Análisis estratificado de la puntuación de la escala: riesgo, riesgo ajustado y probabilidad de supervivencia

\begin{tabular}{|c|c|c|c|c|}
\hline Variable & Vivos 183 & Muertos 44 & HR (IC-95\%)* & Valor de $\mathrm{p}$ \\
\hline Esteroides prenatales & $82(45 \%)$ & $17(39 \%)$ & $0,7(0,1-2,5)$ & 0,6 \\
\hline $\begin{array}{l}\text { Apgar al minuto } 5 \\
\quad 9-10 \\
7-8 \\
<6\end{array}$ & $\begin{array}{c}90(49 \%) \\
77(42 \%) \\
16(9 \%)\end{array}$ & $\begin{array}{c}6(13 \%) \\
22(50 \%) \\
16(37 \%)\end{array}$ & $\begin{array}{c}- \\
1,3(0,4-13) \\
2,7(1,4-5,2)\end{array}$ & $\begin{array}{c}0,3 \\
\mathbf{0 , 0 5}\end{array}$ \\
\hline $\begin{array}{l}\text { Peso al nacer }(\mathrm{g}) \\
\quad>2500 \\
1500-2499 \\
<1499\end{array}$ & $\begin{array}{l}49(27 \%) \\
86(47 \%) \\
48(26 \%)\end{array}$ & $\begin{array}{l}8(18 \%) \\
11(25 \%) \\
25(56 \%)\end{array}$ & $\begin{array}{c}- \\
0,8(0,4-2) \\
4,7(1,2-9)\end{array}$ & $\begin{array}{c}0,4 \\
\mathbf{0 , 0 1}\end{array}$ \\
\hline $\begin{array}{l}\text { Lactato }(\mathrm{mmol} / \mathrm{L}) \\
\quad<1 \\
\quad 1,1-3,9 \\
\quad>4\end{array}$ & $\begin{array}{l}99(54 \%) \\
38(21 \%) \\
46(25 \%)\end{array}$ & $\begin{array}{c}3(7 \%) \\
7(15 \%) \\
34(78 \%)\end{array}$ & $\begin{array}{c}- \\
0,4(0,1-3) \\
5,6(1,2-7)\end{array}$ & $\begin{array}{c}0,6 \\
\mathbf{0 , 0 3}\end{array}$ \\
\hline $\begin{array}{l}\text { a/A } \\
\quad>0,6 \\
\quad 0,59-0,26 \\
\quad<0,25\end{array}$ & $\begin{array}{c}48(26 \%) \\
16(9 \%) \\
19(65 \%)\end{array}$ & $\begin{array}{c}2(5 \%) \\
9(20 \%) \\
33(75 \%)\end{array}$ & $\begin{array}{c}- \\
1,2(0,7-1,7) \\
1,8(0,9-4)\end{array}$ & $\begin{array}{l}0,07 \\
0,06\end{array}$ \\
\hline $\begin{array}{c}\mathrm{P}\left(\mathrm{Ao}_{2}-\mathrm{aO}_{2}\right) \\
<100 \\
100-299 \\
>300\end{array}$ & $\begin{array}{c}9(5 \%) \\
165(90 \%) \\
9(5 \%)\end{array}$ & $\begin{array}{c}2(5 \%) \\
8(18 \%) \\
34(77 \%)\end{array}$ & $\begin{array}{c}- \\
0,2(0,01-0,7) \\
2,5(1,5-10)\end{array}$ & $\begin{array}{c}0,05 \\
0,001\end{array}$ \\
\hline $\begin{array}{l}\text { Plaquetas }(\mathrm{cel} / \mathrm{uL}) \\
\quad>150000 \\
50000-149000 \\
<49000\end{array}$ & $\begin{array}{c}143(78 \%) \\
35(19 \%) \\
5(3 \%)\end{array}$ & $\begin{array}{l}5(12 \%) \\
17(38 \%) \\
22(50 \%)\end{array}$ & $\begin{array}{c}- \\
1,6(0,4-15) \\
6,3(2-17)\end{array}$ & $\begin{array}{c}0,7 \\
\mathbf{0 , 0 0 1}\end{array}$ \\
\hline $\begin{array}{l}\text { Glucosa sérica }(\mathrm{mg} / \mathrm{dl}) \\
\quad<126 \\
127-200 \\
>201\end{array}$ & $\begin{array}{l}114(62 \%) \\
40(22 \%) \\
29(16 \%)\end{array}$ & $\begin{array}{c}8(19 \%) \\
25(56 \%) \\
11(25 \%)\end{array}$ & $\begin{array}{c}- \\
2,3(1,2-6) \\
1,2(0,7-13)\end{array}$ & $\begin{array}{c}\mathbf{0 , 0 5} \\
0,4\end{array}$ \\
\hline $\begin{array}{l}\text { Escala EMN-9 Mex } \\
\text { De } 1 \text { a } 7 \\
\text { De } 8 \text { a } 11 \\
\text { De } 12 \text { a } 15 \\
\text { De } 16 \text { a } 18\end{array}$ & $\begin{array}{c}50(27 \%) \\
80(44 \%) \\
46(25 \%) \\
7(4 \%)\end{array}$ & $\begin{array}{c}1(2 \%) \\
4(10 \%) \\
22(50 \%) \\
17(38 \%)\end{array}$ & $\begin{array}{c}- \\
2,4(0,2-21) \\
18(2,4-133) \\
85(11-102)\end{array}$ & $\begin{array}{c}- \\
0,4 \\
\mathbf{0 , 0 0 5} \\
<\mathbf{0 , 0 0 0 1}\end{array}$ \\
\hline
\end{tabular}

* El guión (-) representa la categoría de referencia.

HR: razón de riesgo.

Tabla 4. Validez de la puntuación de la Escala de Mortalidad Neonatal-9 México dividida en cuatro categorías

\begin{tabular}{lccccccc}
\hline Puntuación & Sensibilidad & Especificidad & VP+ & VP- & LR+ & LR- & AUC \\
\hline De 1 a 7 & $2 \%$ & $73 \%$ & $2 \%$ & $76 \%$ & $0,08(0,01-0,5)$ & $1,34(1,22-1,49)$ & $0,50(0,1-0,7)$ \\
De 8 a 11 & $9 \%$ & $56 \%$ & $5 \%$ & $72 \%$ & $0,21(1,3-1,8)$ & $1,6(1,4-1,7)$ & $0,75(0,63-0,89)$ \\
De 12 a 15 & $50 \%$ & $75 \%$ & $32 \%$ & $86 \%$ & $1,9(1,4-2,8)$ & $0,67(0,5-0,9)$ & $0,92(0,88-0,94)$ \\
De 16 a 18 & $40 \%$ & $99 \%$ & $71 \%$ & $90 \%$ & $10(4,3-23)$ & $0,6(0,4-0,8)$ & $0,95(0,9-0,97)$ \\
\hline
\end{tabular}

VP: valor predictivo.

LR: razón de verosimilitud.

AUC: área bajo la curva. 
en 2001: SNAP-II y SNAPPE II. ${ }^{8}$ Esta última, a diferencia de nuestra escala, se realizó en 25429 neonatos en cuatro centros hospitalarios, con un área bajo la curva de 0,91, sin incluir a neonatos atendidos en la UCIN.

La segunda versión del CRIB ${ }^{32}$ se realizó en el tiempo posterior a la difusión de la aplicación del agente tensoactivo. Incluyó neonatos atendidos en las unidades de cuidados intensivos, pero está diseñada para pacientes con peso menor de 1500 g, en comparación con la nuestra, que tomó en cuenta a recién nacidos de cualquier peso. El CRIB II se ha aplicado en distintos hospitales y reportó un área bajo la curva de entre 0,86 y 0,9.33

En México, en 2001, ${ }^{34}$ se publicó una escala metodológicamente construida con semejanzas a este estudio. Sin embargo, los neonatos en los que se validaron los datos fueron obtenidos de tres centros de referencia del país donde no se atienden nacimientos y el abordaje inicial pudo haber alterado el pronóstico de los sujetos incluidos. Estas condiciones afectan la validez externa de dicha escala, además de que las variables como sepsis y falla cardíaca pueden variar en su identificación de acuerdo a quién las califique. Su interpretación se basa en la sustitución del valor del coeficiente $\beta$ de cada variable en la fórmula de la regresión logística, lo que puede retrasar y dificultar su aplicación en la práctica clínica diaria. Cuenta con un área bajo la curva de 0,86 y no hay otras publicaciones que refieran haberla utilizado o validado.

La escala NEOCOSUR, creada a partir de un esfuerzo multicéntrico en países latinoamericanos, se validó en una población de más de 1800 neonatos de muy bajo peso al nacer, conformada por seis variables pronósticas (peso y edad gestacional, Apgar al primer minuto, malformaciones congénitas, uso de esteroides prenatales y la edad materna) con un área bajo la curva de 0,85 , con la ventaja de poder ser empleada para otros desenlaces además de mortalidad, como displasia broncopulmonar y hemorragia intraventricular. ${ }^{35}$ Comparada frente a las otras escalas, presenta un mejor poder predictivo para mortalidad. ${ }^{8}$

La EMN-9 Mex, por lo tanto, se trata de una escala predictora de mortalidad, de sencilla aplicación e interpretación, que puede emplearse en neonatos independientemente del peso al nacer, que introduce otras variables que no han sido utilizadas en otras escalas.

Las debilidades radican en que el tamaño de la muestra y la población diana son limitados a un

\section{Figura 2. Curva de supervivencia por categoría de acuerdo con la Escala de Mortalidad Neonatal-9 México (EMN-9Mex)}

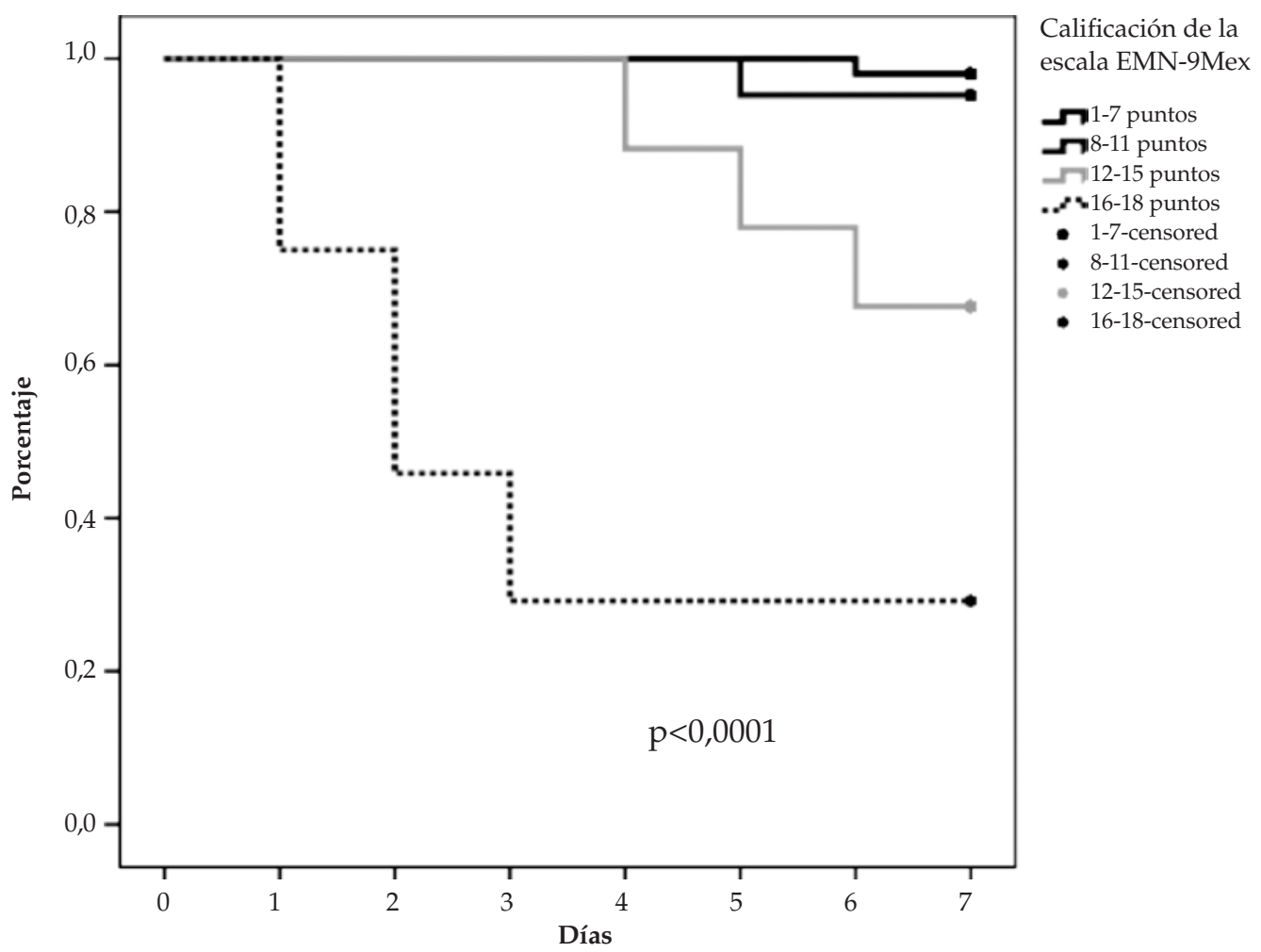


solo centro; el manejo terapéutico está restringido a los recursos institucionales. Los neonatos elegidos fueron exclusivamente atendidos en la Unidad de Cuidados Intensivos y el poder pronóstico de la escala se limita a la mortalidad en la etapa neonatal temprana.

\section{CONCLUSIONES}

La EMN-9 Mex es una propuesta novedosa validada para neonatos de todos los pesos, sustentada en variables que abarcan un espectro amplio de enfermedades que ocasionan la mortalidad neonatal. Amerita que se valide en una población más amplia de neonatos, en otros centros hospitalarios donde se involucre palpablemente el efecto que tiene en los prematuros, prematuros tardíos, término y postérmino.

\section{REFERENCIAS}

1. Shiffman J. Issue attention in global health: the case of newborn survival. Lancet 2010;375(9730):2045-9.

2. Oestergaard MZ, Inoue M, Yoshida S, Mahanani WR, et al. Neonatal mortality levels for 193 countries in 2009 with trends since 1990: a systematic analysis of progress, projections, and priorities. PLoS Med 2011;8(8):e1001080.

3. Martines J, Paul VK, Bhutta ZA, KoblinskyM, etal. Neonatal survival: a call for action. Lancet 2005;365(9465):1189-97.

4. Murguía SM, Lozano R, Santos JI. Mortalidad perinatal por asfixia en México: problema prioritario de salud pública por resolver. Bol Med Hosp Infant Mex 2005;62(5):375-83.

5. Záyago-Espinosa M. Utilidad de dos escalas de gravedad como factor de predictivo de mortalidad en neonatos pretérminos. Rev Sanid Milit Mex 2006;60(4):243-7.

6. Gagliardi L, Cavazza A, Brunelli A, Battaglioli M, et al. Assessing mortality risk in very low birthweight infants: a comparison of CRIB, CRIB-II, and SNAPPE-II. Arch Dis Fetal Neonatal Ed 2004;89(5):F419-22.

7. Maier RF, Rey M, Metze BC, Obladen M. Comparison of mortality risk: a score for very low birthweight infants. Arch Dis Child Fetal Neonatal Ed 1997;76(3):F146-50.

8. Marshall G, Tapia JL, D'Apremont I, Grandi C, et al. A new score for predicting neonatal very low birth weight mortality risk in the NEOCOSURSouth American Network. J Perinatol 2005;25(9):577-82.

9. Aguilar-Barojas S. Fórmulas para el cálculo de la muestra en investigaciones de salud. Salud en Tabasco 2005;11(12):333-8.

10. Casey BM, Mclntire DD, Leveno KJ. The continuing value of Apgar score for the assessment of newborn infants. $N$ Eng J Med 2001;344(7):467-71.

11. Lawn CJ, Weir FJ, McGuire W. Base administration or fluid bolus for preventing morbidity and mortality in preterm infants with metabolic acidosis. Chochrane Database Syst Rev 2005;2:CD003215.

12. García HJ, Aparicio-de la Luz S, Franco-Gutiérrez M, González-Lara D, et al. Factores pronósticos de asociados a mortalidad en recién nacidos con hernia diafragmática congénita. Gac Med Mex 2003;139(1):7-14.

13. Yasmin S, Osrin D, Paul E, Costello A. Neonatal mortality of low-birth-weight infants in Bangladesh. Bull World Health Organ 2001;79(7):608-14.

14. Kao LS, Morris BH, Lally KP, Stewart CD, et al. Hyperglycemia and morbidity and mortality in extremely low birth weight infants. J Perinatol 2006;26(12):730-6.
15. Vincent JL, Yagushi A, Pradier O. Platelet function in sepsis. Crit Care Med 2002;30(5 Suppl):S313-7.

16. Fernández HG, Vieira AA, Barbosa AD. The correlation between plasma lactate concentrations and early neonatal mortality. Rev Bras Ter Intensiva 2012;24(2):184-7.

17. Mathur NB, Garg P, Mishra TK. Predictors of fatality in neonates requiring mechanical ventilation. Indian Pediatr 2005;42(7):645-51.

18. Dimitriou G, Fouzas S, Giannakopoulos I, Papadopoulos VG, et al. Prediction of respiratory failure in late-preterm infants with respiratory distress at birth. Eur J Pediatr 2011;170(1):45-50.

19. Levit O, Bhandari V, Li FY, Shabanova V, et al. Clinical and laboratory factors that predict death in very low birth weight infants presenting with late-onset sepsis. Pediatr Infect Dis J 2014;33(2):143-6.

20. Richardson DK, Corcoran JD, Escobar GJ, Lee SK. SNAP-II and SNAPPE-II: Simplified newborn illness severity and mortality risk scores. J Pediatr 2001;138(1):92-100.

21. McCormick MC. The contribution of low birth weight to infant mortality and childhood morbidity. $N$ Eng J Med 1985;312(2):82-90.

22. Unterscheider J, O'Donoghue K, Daly S, Geary MP, et al. Fetal growth restriction and the risk of perinatal mortalitycase studies from de multicentre PORTO study. BMC Pregnancy Childbirth 2014;14:63.

23. Alexander GR, de Caunes F, Hulsey TC, Tompkins ME, et al. Validity of postnatal assessments of gestational age: a comparison of the method of Ballard et al. and early ultrasonography. Am J Obstet Gynecol 1992;166(3);891-5.

24. Yau KI, Hsu CH. Factors affecting the mortality of sick newborns admitted to intensive care units. Acta Paedtr Taiwan 1999;40(2):75-82.

25. Horbar JD. A calculator program for determining indices of neonatal respiratory distress syndrome severity. Am J Perinatol 1987;4(1):20-3.

26. Mendoza-Domínguez S, Zavala-Mendoza A, LópezTamanaja NL, Rodríguez-Zepeda JJ, et al. Índices de oxigenación en recién nacidos en estado crítico. Rev Mex Pediatr 1999;66(1):14-7.

27. Varkilova L,Slancheva B, Emilova Z, Nikolov A, et al. Blood lactate measurements as a diagnostic and prognostic tool after birth asphyxia in newborn infants with gestational age $>$ or $=34$ gestational weeks. Akush Ginekol (Sofiia) 2013;52(3):36-43. Bulgaria.

28. Bauman ME, Cheung PY, Massicotte MP. Hemostasis and platelet dysfunction in asphyxiated neonates. J Pediatr 2011;158(2 Suppl):e35-9.

29. Beardsall K, VanhaesebrouckS, Ogilvy-Stuart AL, Vanhole $\mathrm{C}$, et al. Prevalence and determinants of hyperglycemia in very low birth weight infants: cohort analyses of the NIRTURE study. J Pediatr 2010;157(5):715-9.

30. Hirshberg E, Larsen G, Van Duker H. Alterations in glucose homeostasis in the pediatric intensive care unit: Hyperglycemia and glucose variability are associated with increased mortality and morbidity. Pediatr Crit Care Med 2008;9(4):361-6.

31. ApgarV.Infant resuscitation. 1957. Conn Med 2007;71(9):553-5.

32. Baumer JH, Wright D, Mill T. Illness severity measured by CRIB score: a product of changes in perinatal care? Arch Dis Child Fetal Neonatal Ed 1997;77(3):F211-5.

33. Sarquis AL, Miyaki M, Cat MN. Aplicação do escore CRIB para avaliar o risco de mortalidade neonatal. J Pediatr (Rio J) 2002;78(3):225-9.

34. García H, Villegas-Silva R, Villanueva-García D, GonzálezCabello $\mathrm{H}$, et al. Validation of a prognostic index in the critically ill newborn. Rev Investig Clin 2000;52(4):406-14.

35. Tavosnanska J, Carreras IM, Fariña D, Luchternberg G, et al. Morbimortalidad de recién nacidos con menos de 1500 gramos asistidos en hospitales públicos de la Ciudad de Buenos Aires. Arch Argent Pediatr 2012;110(5):394-403. 